\title{
622 - High-precision Cryogenic Correlative Light and Electron Microscopy Using Two Independent Alignment Features and an Easy-to-use Toolkit to Study Virus- infected Mammalian Cells
}

\author{
Jae Yang, Bryan Sibert, Matthew Larson, Samantha Shrum and Elizabeth Wright \\ University of Wisconsin-Madison, Madison, Wisconsin, United States
}

Cryogenic correlative light and electron microscopy (cryo-CLEM) has proven to be a powerful tool to study in situ the high-resolution structures of macromolecules and biological events such as virus infection, in their native states. The fluorescent labeling and imaging of objects enables one to rapidly identify the target and orient on its spatial localization within a complex matrix. Cryo-EM and cryo-ET have become essential for extending our understanding of the mechanisms of viral infection and the native structures of pleomorphic viruses and virus-infected cells in plunge-frozen specimens. Over the past few years, exciting developments have greatly advanced this field [1-4]. Despite technological advances, cryoCLEM suffers from low localization precision and remains to be a system-dependent process.

A number of research groups have developed tools to facilitate the correlation steps between LM and EM imaging platforms. EM grids marked with numbers and letters (i.e., Finder grids) aid in the first steps of rough correlation. Finder grids support an approximate relocation of the region of interest (ROI) in EM at a lower-resolution (micrometer-level) and larger object size scale, such as the identification of certain parts of cells or squares on the EM grid [3,5]. Additional fiducial markers applied to a sample, such as fluorescent electron-dense microspheres, has supported finer-scale correlations to approximately $100 \mathrm{~nm}$ $[5,6]$. A less common method is to use sample support features, including holes and imperfections in the carbon film, to achieve a marker-free correlative alignment [7]. However, in all cases, the distribution of markers across the entire grid needs to be relatively uniform for alignments to be reliable. Moreover, bent, warped, or crinkled frozen grids and uneven ice-thickness that may be introduced by poor handling and the freezing process hinder the detection of the holes in the carbon films. This could lead to a poor relocation of the same hole in both maps. Here, we present a workflow implementing (1) the Finder grid to aid the rough correlation of the fluorescence map to the low magnification TEM map, (2) the autoidentification of hole centers in the carbon film, in combination with a random distribution of fluorescent microspheres, for an accurate correlation and transfer of coordinates between the two maps. To improve and accelerate the correlation efficiency, we have developed a user-friendly correlation toolkit applicable to both on-the-fly and post-acquisition high-accuracy correlative coordinate registration, adaptable to many microscope systems. The workflow implementation was demonstrated at room temperature (RT) using microspheres deposited on Finder grids, and under cryo-conditions by visualizing cells infected with respiratory syncytial virus (RSV).

Quantifoil gold Finder grids were coated with an extra layer of carbon for stabilization and glow discharged for improved hydrophilicity. The grids were subsequently coated with a diluted solution of fluorescent microspheres (Invitrogen) to assist fine correlation. For cryo-CLEM, HeLa cells were grown on the grids and infected by recombinant virus strain RSV rA2-mK+ that has a far-red fluorescent reporter gene [8]. Subsequently, native immunogold labeling of RSV glycoprotein F was carried out 24-hr postinfection, as described previously [9]. The grids were vitrified by plunge-freezing. The cryo-fluorescent microscopy was performed with a Leica EM Cryo-CLEM system [10]. Then the same grid was visualized under cryo-conditions with a ThermoFisher Tecnai TF30 equipped with a Gatan K2 summit direct electron detector camera. Auto-identification of hole centers on both fluorescence microscopy and TEM maps was performed following a similar procedure described in [7]. The correlative toolkit developed in our lab and 
SerialEM software [11] were used to perform both rough and fine registration transfers between the two maps.

To obtain an estimate of the alignment accuracy, we averaged individual prediction errors following a similar procedure developed for fiducial-based alignments [5,6]. As shown in Figure 1, in $\sim 30$ highmagnification images, the predicted location of the fluorescence signal is clearly associated with a virus that is visible in the image. We measured the deviation of the predicted coordinate from the center of the targeted microspheres or viral particles (Figure 2). This standard deviation of the measurements is $6 \mathrm{~nm}$ and $7 \mathrm{~nm}$ in $\mathrm{X}$ and Y for fluoro-microsphere-deposited Finder grids imaged at RT, and 32 and $38 \mathrm{~nm}$ for immuno-labeled RSV-infected cell Finder grids under cryo-conditions.

We found that the implementation of the new workflow supports an easy and accelerated localization of ROI and targets with a relocation precision of less than $90 \mathrm{~nm}$ in average. This workflow, including our easy-to-use correlative toolkit, is applicable to existing cryo-CLEM systems and may be adapted for use with both room temperature and cryo-super resolution correlative microscopy [12].
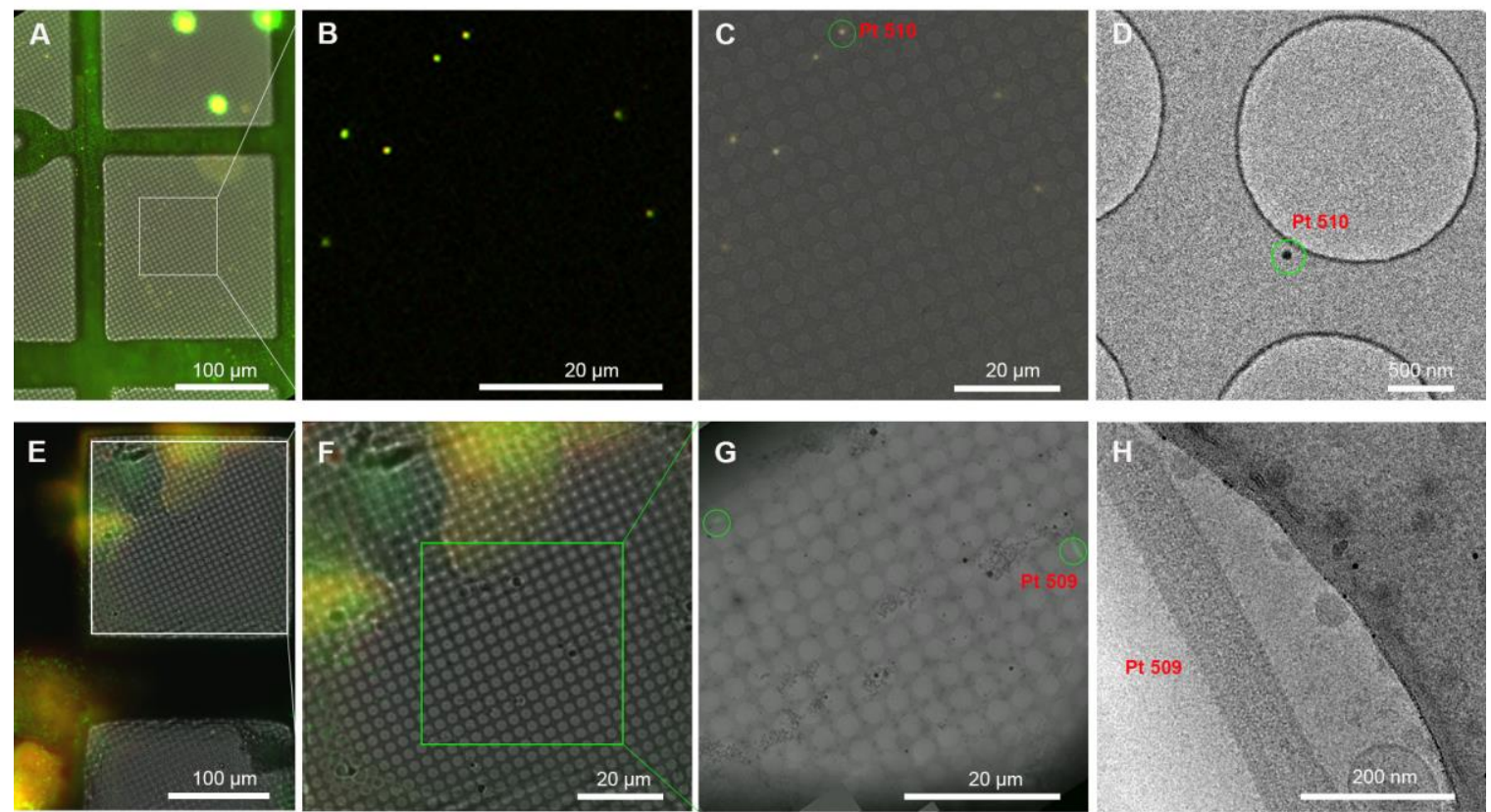

Figure 1. Square overviews of the fluorescent microsphere coated RT Finder grid (A) and labeled RSVinfected HeLa cell cryo-grid (E). The microspheres appear in yellow. Infected cells signal red and viral particles are green. (B, F) Zoom into the white marked ROI. (C, G) Superpositions of the TEM montage of the selected area and the transformed fluorescent map after correlation. (D, H) High mag images collected at the marked point of interest, Pt 510 for the target fluorescent microsphere, and Pt 509 for the target RSV particle. 

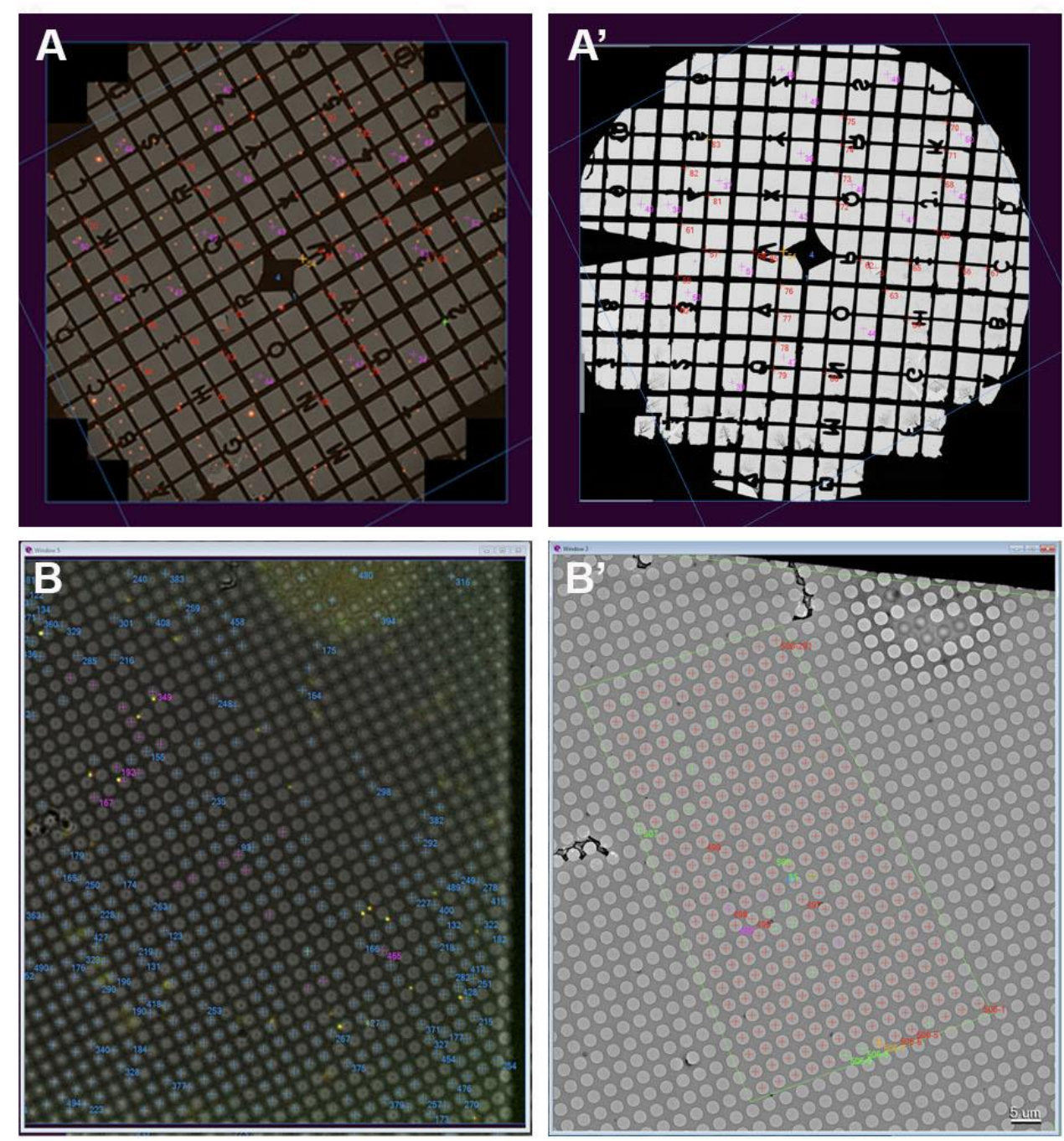

D
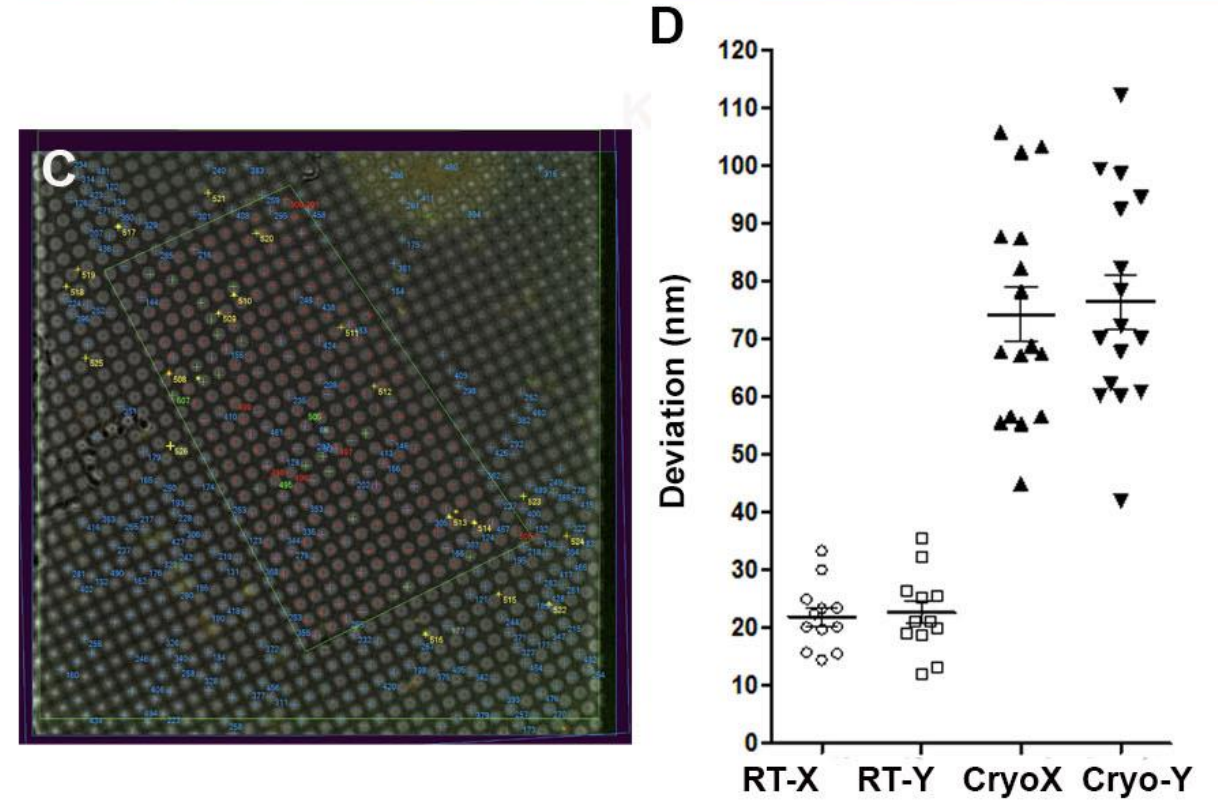
Figure 2. Screenshots of the procedure to set up the high-precision correlation scheme, as demonstrated in RT Finder grids (A-C). (A-A') Rough correlation of the fluorescent map and low mag TEM montage using the Finder grid's letters and large land markers. (B-B') High-precision finer correlation through auto-identification of hole centers on both two maps. (C) Successful transform after correlative registration. (D) Distribution of the deviation of the predicted coordinate from the center of the targeted object for each coordinate axis, at both RT and cryo-conditions.

\section{References}

(1) Wolff, G.; Biol. Cell 2016, 108 (9), 245-258.

(2) Schorb, M.; J. Struct. Biol. 2017, 197 (2), 83-93.

(3) Fu, X.; Sci. Rep. 2019, 9 (1), 19207. https://doi.org/10.1038/s41598-019-55766-8.

(4) Schorb, M.; Nat. Methods 2019, 16 (6), 471-477.

(5) Schellenberger, P.; Ultramicroscopy 2014, 143, 41-51.

(6) Kukulski, W.; J. Cell Biol. 2011, 192 (1), 111-119.

(7) Anderson, K. L.; J. Struct. Biol. 2018, 201 (1), 46-51.

(8) Hotard, A. L.; Virology 2012, 434 (1), 129-136.

(9) Yi, H.; J. Histochem. Cytochem. 2015, 63 (10), 780-792.

(10) Hampton, C. M.; Nat. Protoc. 2016, 12 (1), 150-167.

(11) Mastronarde, D. N. J. Struct. Biol. 2005, 152 (1), 36-51.

(12) This research was supported in part by funds from the University of Wisconsin, Madison and the National Institutes of Health (R01GM114561 and R01GM104540-03S1) to E.R.W. The authors gratefully acknowledge use of facilities and instrumentation at the UW-Madison Wisconsin Centers for Nanoscale Technology (wcnt.wisc.edu) partially supported by the NSF through the University of Wisconsin Materials Research Science and Engineering Center (DMR-1720415). A portion of this research was supported by NIH grant U24GM129547 and performed at the PNCC at OHSU and accessed through EMSL (grid.436923.9), a DOE Office of Science User Facility sponsored by the Office of Biological and Environmental Research. 